\title{
Pharmacists without Access to the EHR: Practicing with One Hand Tied Behind Our Backs
}

Deeatra S. Craddock, PharmD, BCACP; Ronald G. Hall 2nd, PhamD, MSCS

Texas Tech University Health Sciences Center, Jerry H. Hodge School of Pharmacy, Department of Pharmacy Practice

\begin{abstract}
Pharmacists are the most accessible healthcare professionals to the public, yet have the least amount of information from the electronic health record available to them. This lack of information makes ensuring that patients are receiving proper medications and monitoring for efficacy and safety a challenge, if not impossible in some situations. Having access to a national electronic health record would provide pharmacists with this needed information to truly engage with prescribers as fellow clinical experts in the field. Sharing prescription information for non-controlled substances would also decrease the likelihood of a patient receiving duplicative therapy from two prescribers or pharmacies that may not know what the other is doing. There are already examples of successful national data sharing including the Prescription drug Monitoring Program for controlled substances as well as the Veterans Affairs healthcare system. Therefore, our profession needs to push for nationwide access to patient electronic health records, which includes all healthcare providers. This will facilitate the inclusion of pharmacists in the optimization of the care of patients who need our expertise in managing their medication regimens as well as build better relationships with prescribing providers.
\end{abstract}

There is an adage in oncology that goes "No meat, no treat" which means that oncologists refuse to treat a suspected cancer without having adequate information to make a diagnosis and treatment plan. Yet, in pharmacies across the United States, the exact opposite happens. A prescription is sent to a pharmacy and the pharmacist is supposed to dispense the prescription safely and efficiently. The only information available to the pharmacist is the information in the prescription and any previous information in the pharmacy computer system (ie, demographics, insurance, prescriptions previously filled at the pharmacy). Healthcare providers that see established patients would be aghast at the lack of information available to community pharmacists to make decisions with a patient. Yet, this is accepted as the standard of care for community pharmacies across the United States. The lack of information is partly because the United States of America does not have a national EHR. The World Health Organization reports that $>50 \%$ of upper-middle- and highincome countries had adopted a national EHR by $2016 .{ }^{1}$ The report also noted that $72 \%$ of World Health Organization member states with a national EHR reported integration of the information system with pharmacy. The current system in the US results in providers who can transmit prescriptions to pharmacies, but pharmacists having no reasonable means of having all of the needed electronic health record (EHR) data to verify the appropriateness of the prescription. Previous data suggests that $39 \%$ of medication therapy management (MTM) visits or consults could be completed without contacting the patient or prescriber if the pharmacist simply had access to the EHR to view the progress notes, diagnoses, labs, and allergies. ${ }^{2}$

Corresponding author: Deeatra S. Craddock, PharmD, BCACP Assistant Professor

Texas Tech University Health Sciences Center

Jerry $\mathrm{H}$. Hodge School of Pharmacy

Department of Pharmacy Practice

5920 Forest Park Rd, Suite 500, Dallas, TX 75235

Email: deeatra.craddock@ttuhsc.edu
The United States has examples of successful closed-loop systems including the Veterans Affairs (VA) and Kaiser Permanente. The VA has shown the benefits of being able to take care of patients inside of a system where all of the healthcare providers have equal access to the information in a patient's electronic health record (EHR). ${ }^{3}$ Kaiser is an example of a private closed-loop system in the US and has been able to demonstrate its efficiency when compared to the National Health Service in England, another closed-loop system. ${ }^{4,5}$ The vast majority of Americans are in open healthcare systems where they have the freedom to choose any medical provider they want regardless of whether that provider has access to the patient's previous medical history. These open systems require more proactive coordination of care to help optimize the safety and efficacy of both non-pharmacologic and pharmacologic treatment and monitoring plans. There are numerous reports in the literature about failures in the transitions of care as well as their associated costs and outcomes. ${ }^{6-8}$ This has led to a greater emphasis of involving pharmacists in transitions of care to try to minimize the risk of miscommunications (or a lack of communication) that can occur when a patient is transitioning from one setting to another. ${ }^{9-11}$

Community pharmacists are consistently recognized as the most accessible professional in healthcare. ${ }^{12}$ Why are these valuable professionals denied access to the patient's health record? How can a pharmacist reasonably ascertain that a prescription is appropriate for a patient without knowledge of a diagnosis or indication? Time is lost by providers, pharmacists, and possibly patients when pharmacists present interventions that are not accurate or information in the EHR alters the original recommendation. ${ }^{13} \mathrm{~A}$ previous study has shown that $25 \%$ of drug therapy problems (DTP) are invalid upon pharmacist EHR review. Of the recommendations that were viewed as valid, EHR review influenced the confidence level of $45 \%$ of the interventions. ${ }^{14}$ 
It is easy to point the finger at the numerous EHR platforms available for medical practices to use. However, these practices have no issues in being able to e-prescribe a prescription with any pharmacy in the country and e-prescriptions represented $80 \%$ of all prescriptions filled in $2019 .{ }^{15}$ Pharmacies are privy to and routinely employ the use of the Prescription Monitoring Program (PMP) for their state regardless of whether they are a chain or an independent. The National Association of Boards of Pharmacy's PMP InterConnect program allows pharmacies to connect to any of the 48 states that are participants. ${ }^{16}$ Access to relevant lab values, such as an INR or serum creatinine $(\mathrm{SCr})$ could help pharmacists make clinical judgements about the proper dosage of medications, appropriateness, and other factors that promote the goals of therapy. In addition, the benefits of the pharmacist having EHR access can yield fruitful results on diabetes and transition of care outcomes (Table 1). ${ }^{17-}$ 19 So, technological issues can be overcome if giving all providers equal access to a patient's medical record is viewed as a priority.

Many states allow or compensate community pharmacists for patient care activities that do not involve drug dispensing, including medication therapy management, collaborative practice agreements, and vaccine administration. 20,21 Pharmacists are also a valuable resource in helping with medication reconciliation as well as an assessment of a patient's allergy history. ${ }^{22-25}$ The patient often has to get this information back to their Primary Care Provider to ensure their EHR is updated. Specialty physician groups also need a mechanism to be able to send patient data back and forth with a patient's PCP if both physicians are not in the same medical group. Similarly, pharmacists may not know what other prescriptions for non-controlled substances a patient has at other pharmacies. This lack of information is concerning in terms of both safety and potential for medication misuse. Other areas where patient data is likely to fall through the cracks include retail clinics, urgent care visits, and the purchase of nonprescription medications or herbals.

There are some potential concerns associated with the implementation of a national EHR. First and foremost, there would be concerns about patient confidentiality and/or data breaches. However, we could learn from the experience in Australia as well as other countries that have implemented a national EHR. As of 2019, only 10\% of Australians have opted out of their national EHR. ${ }^{26}$ There are also examples in the United States of national access to patient records within a given company (ie, VA, Kaiser, chain pharmacies). Kansas is a specific example of a state that utilizes a health interface that has empowered community pharmacists to help decrease hospital readmissions. ${ }^{27}$

Federal protections of the network, as well as stiffer federal penalties for breaches of confidentiality, would be needed. An efficient way to authorize access to a patient's EHR is needed to help be a safeguard. Furthermore, non-prescription drugs and herbals would still not be captured at the purchase level if they were not purchased at a pharmacy. Getting this information at the purchase level would require linking credit or debit card purchases. This approach still might not be linked with the correct patient[s] taking them and would not capture cash purchases.

Community pharmacies are a key healthcare resource in America, but community pharmacists have been practicing with one hand tied behind their backs for too long. It is time for these valuable healthcare providers to have access to make more informed decisions to help optimize the safety and effectiveness of the treatment plan for their patients. Having EHR access would allow pharmacists to build better relationships with prescribing providers and offer even better recommendations based on a more complete view of the patient's history. A national EHR is a necessary step to help minimize the issues with transitions in care and to allow all healthcare providers access to the same information to make informed decisions with their patients.

Author contribution statement: Both authors (Craddock and Hall): Conceptualization, Data curation, Formal analysis, Methodology; Project administration, Resources; Software; Supervision, Roles/Writing - original draft; Writing - review \& editing

Not applicable: Funding acquisition; Investigation; Validation; Visualization

Funding support: This commentary did not receive any specific grant from funding agencies in the public, commercial, or not-for-profit sectors.

Disclosures of conflicts of interest: DC: Dr. Craddock declares no relevant conflicts of interest or financial relationships. $\mathrm{RH}$ : Merck (grant funding)

\section{References}

1. https://www.who.int/gho/goe/electronic health records/ en/.

2. Roberts MF, Reeves K, Divine H. Community pharmacists' lack of access to health records and its impact on targeted MTM interventions. J Am Pharm Assoc (2003). 2019;59(4S):S81-S84.

3. O'Hanlon C, Huang C, Sloss E, et al. Comparing VA and NonVA Quality of Care: A Systematic Review. Journal of General Internal Medicine. 2017;32(1):105-121.

4. Feachem RGA. Getting more for their dollar: a comparison of the NHS with California's Kaiser Permanente Commentary: Funding is not the only factor Commentary: Same price, better care Commentary: Competition made them do it. BMJ. 2002;324(7330):135-143. 
5. Ham C. Hospital bed utilisation in the NHS, Kaiser Permanente, and the US Medicare programme: analysis of routine data. BMJ. 2003;327(7426):1257-1250.

6. Forster AJ, Murff HJ, Peterson JF, Gandhi TK, Bates DW. Adverse drug events occurring following hospital discharge. Journal of General Internal Medicine. 2005;20(4):317-323.

7. Solet DJ, Norvell JM, Rutan GH, Frankel RM. Lost in translation: challenges and opportunities in physician-tophysician communication during patient handoffs. Acad Med. 2005;80(12):1094-1099.

8. Medicare Payment Advisory Commission, Report to the Congress: Reforming the Delivery System, Washington, D.C.: MedPAC, June 2008.

9. Brauner MS, Accursi ML, Cantillo SL, Baxter MV, Burant CJ. Project EVADE: Evaluating the effects of a pharmacist-run transitions of care clinic on hospital readmissions. J Am Pharm Assoc (2003). 2020;60(3):503-508.

10. Feldmann JD, Otting RI, Otting CM, Witry MJ. A community pharmacist-led service to facilitate care transitions and reduce hospital readmissions. J Am Pharm Assoc (2003). 2018;58(1):36-43.

11. Novak CJ, Hastanan S, Moradi M, Terry DF. Reducing unnecessary hospital readmissions: the pharmacist's role in care transitions. Consult Pharm. 2012;27(3):174-179.

12. Manolakis PG, Skelton JB. Pharmacists' Contributions to Primary Care in the United States Collaborating to Address Unmet Patient Care Needs: The Emerging Role for Pharmacists to Address the Shortage of Primary Care Providers. American Journal of Pharmaceutical Education. 2010;74(10):S7.

13. Smith M, Sprecher B. Pharmacy communications with physician offices to clarify prescriptions. J Am Pharm Assoc (2003). 2017;57(2):178-182.

14. van Lint JA, Sorge LA, Sorensen TD. Access to patients' health records for drug therapy problem determination by pharmacists. J Am Pharm Assoc (2003). 2015;55(3):278-281.

15. https://www.statista.com/statistics/864380/share-of-us-eprescriptions/\#: :text=In\%202019\%2C\%2080\%20percent\% 20of,for\%20controlled\%20substances\%20like\%20opioids.

16. https://nabp.pharmacy/members/pmp-interconnect/.

17. Snyder JM, Ahmed-Sarwar N, Gardiner C, Burke ES. Community pharmacist collaboration with a primary care clinic to improve diabetes care. J Am Pharm Assoc (2003). 2020;60(3S):S84-S90.

18. Bacci JL, Berenbrok LA. Innovative Advances in Connectivity and Community Pharmacist Patient Care Services: Implications for Patient Safety. Pharmacotherapy. 2018;38(8):867-874.

19. Keller ME, Kelling SE, Cornelius DC, Oni HA, Bright DR. Enhancing Practice Efficiency and Patient Care by Sharing Electronic Health Records. Perspect Health Inf Manag. 2015;12:1b.

20. Woodhouse A, Clements E, Keedy C, Presnell A, Schnibben A. Integration and compensation of pharmacists into primary care medical groups. J Am Pharm Assoc (2003). 2019;59(6):886-890.

21. Stergachis A. Strengthening the evidence-base on payment models for pharmacist-provided services. J Am Pharm Assoc (2003). 2019;59(1):5.

22. Mekonnen AB, McLachlan AJ, Brien J-AE. Pharmacy-led medication reconciliation programmes at hospital transitions: a systematic review and meta-analysis. Journal of Clinical Pharmacy and Therapeutics. 2016;41(2):128-144.
23. Kilcup M, Schultz D, Carlson J, Wilson B. Postdischarge pharmacist medication reconciliation: Impact on readmission rates and financial savings. Journal of the American Pharmacists Association. 2013;53(1):78-84.

24. Sigona NS, Steele JM, Miller CD. Impact of a pharmacistdriven beta-lactam allergy interview on inpatient antimicrobial therapy: A pilot project. J Am Pharm Assoc (2003). 2016;56(6):665-669.

25. Bland CM, Jones BM. Pharmacists Filling the Gap Within Penicillin Allergy Assessment and Skin Testing. Clinical Infectious Diseases. 2020.

26. https://www.healthcareitnews.com/news/apac/one-tenaustralians-opt-out-my-health-record-system-adha-says.

27. Fanizza FA, Ruisinger JF, Prohaska ES, Melton BL. Integrating a health information exchange into a community pharmacy transitions of care service. J Am Pharm Assoc (2003). 2018;58(4):442-449. 
Table 1. Pharmacists collaborations with EHR access and outcomes

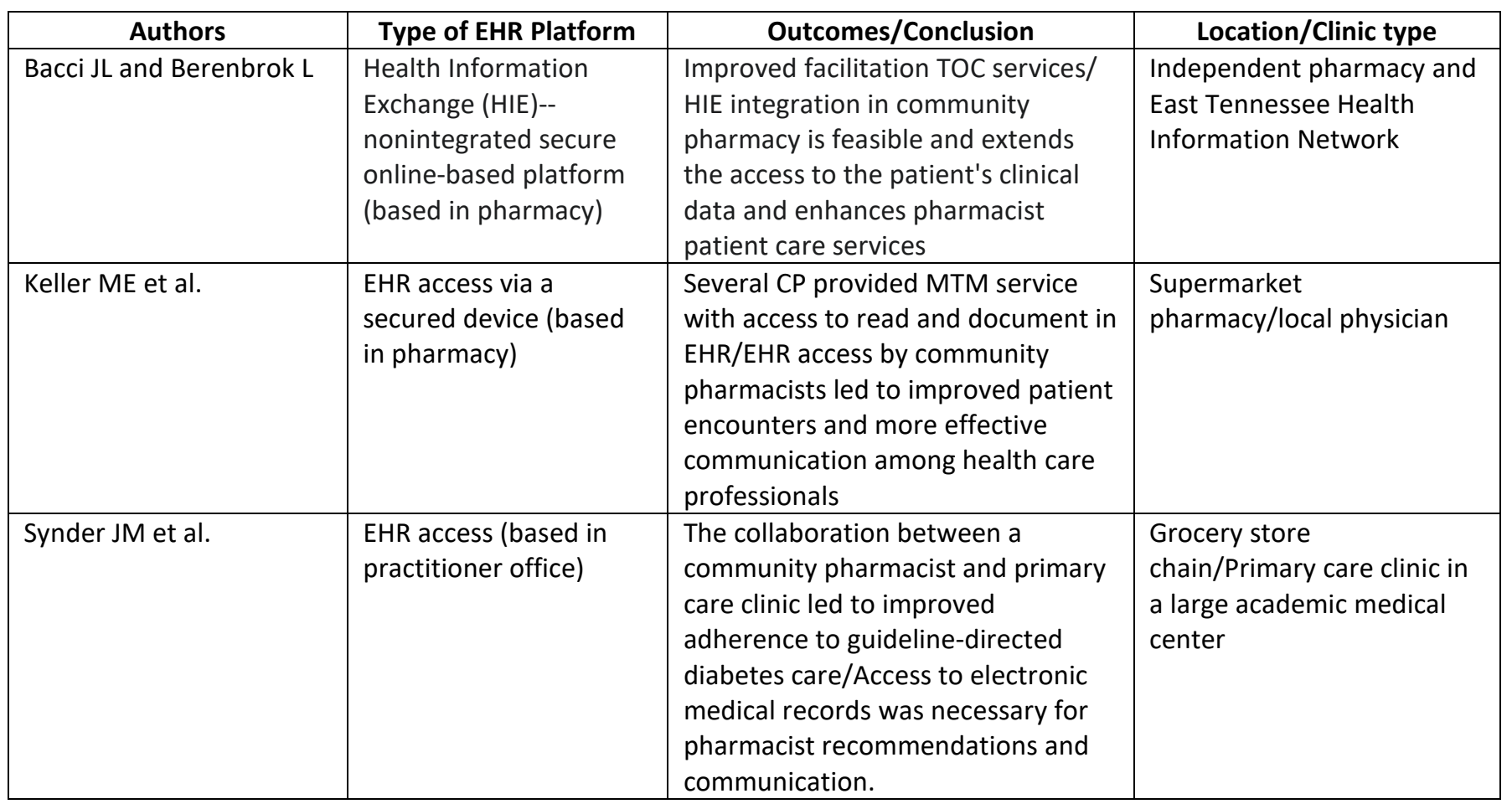

